\title{
Structural Operational Semantics with transitivity rules and execution time
}

\author{
Patricia Peratto \\ psperatto@adinet.com.uy \\ Tecnólogo en Informática \\ FING-CETP.UdelaR-ANEP
}

March 2009

\begin{abstract}
We define an structural operational semantics of the core of an imperative language. It has a measure in the transitions corresponding to the number of steps of evaluation that takes place in the transition (from the point of view of usual complexity theory) and transitivity rules that allow to prove in the theory what is usually proved in the meta-theory.
\end{abstract}

\section{Introduction}

Our semantics has transitions of the form $\Rightarrow^{k}$ where $k$ is the number of steps of evaluation that takes place in the transition.

Rules to measure the time of evaluation of arithmetic expressions and statements for the natural semantics have been given in [3]. To our knowledge, haven't been presented for the structural operational semantics.

This semantics applies to the evaluation of expressions and statements and can allow to measure the complexity of a program at the same time that we derive its construction applying the rules. The semantics is compositional and the measure of time also.

We show our semantics is deterministic and equivalent to the structural operational semantics without transitivity rules. We also show it is equivalent to the natural semantics with exact execution times presented in [3].

The outline of the paper is as follows: in section 2 we present the abstract syntax of the language and we introduce categories for different kind of expressions and for statements; follows in section 3 the semantics of the expressions and the set of rules for the semantics of statements. In section 4 we prove that the semantics is deterministic. In section 5 we present the equivalence with structural operational semantics. In section 6 we present the equivalence with natural semantics with execution times. In section 7 we present conclutions and further work.

\section{Abstract syntax of the language}

We use a syntactic notation based on BNF and we will use parenthesis (not indicated in our BNF) to solve ambiguities and uniquely determine the corresponding parse tree.

We have the following Syntactic Categories and meta-variables ranging over them: 
$n$ will range over numerals, Num

ax will range over arithmetic variables, AVar

$a$ will range over arithmetic expressions, AExp

$b$ will range over boolean expressions, BExp

$S$ will range over statements, $\mathbf{S t m}$

The meta-variables can be primed or subscripted for example $n, n^{\prime}, n_{1}, n_{2}$ all stand for numerals.

\subsection{Abstract syntax for arithmetic variables}

$x::=$ letter $l x$

$l x::=\epsilon \mid$ letter $l x \mid$ digit $l x$

That is, an string of letters and digits beginning with a letter. We assume letter and digit as understood.

\subsection{Abstract syntax for Arithmetic Expressions}

$a::=n|a x| a_{1}+a_{2}\left|a_{1} * a_{2}\right| a_{1}-a_{2}$

\subsection{Abstract syntax for Boolean Expressions}

$b::=$ true $\mid$ false $\left|a_{1}=a_{2}\right| a_{1}<a_{2}|\neg b| b_{1} \vee b_{2} \mid b_{1} \wedge b_{2}$

\subsection{Abstract syntax for Statements}

$S:=$ ax $:=a \mid$ skip $\left|S_{1} ; S_{2}\right|$ if $b$ then $S_{1}$ else $S_{2} \mid$ while $b$ do $S$

\section{Measured semantics of expressions}

We define the following semantic functions:

$A: \mathbf{A e x p} \rightarrow($ State $\rightarrow \mathbf{Z} \times \mathbf{N})$ for arithmetic expressions

$B: \mathbf{B e x p} \rightarrow(\mathbf{T} \times \mathbf{N})$ for boolean expressions

where

$\mathbf{N}$ is the semantic domain of natural numbers,

$\mathbf{Z}$ is the semantic domain of integers,

$\mathbf{T}$ is the semantic domain of truth values,

and

State $=$ AVar $\rightarrow \mathbf{Z}$

We have the following metavariables ranging over semantic categories: $k \in \mathbf{N}$, $z \in \mathbf{Z}, t \in \mathbf{T}$ and $s \in$ State primed or subscripted. 


\subsection{Semantic of Arithmetic Expressions}

We define a transition system with transitions of the form

$<a, s>\rightarrow^{k} z$

We define the following semantic functions

$A(a) s=<z, k>$ if exists $k$ such that $\left\langle a, s>\rightarrow^{k} z\right.$

$\operatorname{Value}(a) s=\operatorname{first}(A(a) s)$

Time $(a) s=\operatorname{second}(A(a) s)$

Transition system:

$<n, s>\rightarrow^{1} n$

$<a x, s>\rightarrow^{1} s(a x)$

$\frac{\left\langle a_{1}, s>\rightarrow^{k_{1}} z_{1} \quad\left\langle a_{2}, s>\rightarrow^{k_{2}} z_{2}\right.\right.}{\left\langle a_{1}+a_{2}, s>\rightarrow^{k_{1}+k_{2}+1} z_{1}+z_{2}\right.}$

$\frac{<a_{1}, s>\rightarrow^{k_{1}} z_{1} \quad\left\langle a_{2}, s>\rightarrow^{k_{2}} z_{2}\right.}{<a_{1} * a_{2}, s>\rightarrow^{k_{1}+k_{2}+1} z_{1} * z_{2}}$

$\frac{<a_{1}, s>\rightarrow^{k_{1}} z_{1} \quad\left\langle a_{2}, s>\rightarrow^{k_{2}} z_{2}\right.}{<a_{1}-a_{2}, s>\rightarrow^{k_{1}+k_{2}+1} z_{1}-z_{2}}$

Theorem 3.1. A, Value and Time are functions.

Given $a$ and $s$, there exists only one $k$ and $z$ such that $\left\langle a, s>\rightarrow^{k} z\right.$.

Proof. By structural induction on $a$.

\subsection{Semantic of Boolean Expressions}

We define a transition system with transitions of the form

$<b, s>\rightarrow^{k} t$

We define the following semantic functions

$B(b) s=<t, k>$ if exists $k$ such that $\left\langle b, s>\rightarrow^{k} t\right.$

$\operatorname{Value}(b) s=\operatorname{first}(B(b) s)$

Time $(b) s=\operatorname{second}(B(b) s)$

Transition system: 


$$
\begin{aligned}
& <\text { true, } s>\rightarrow^{1} \text { true } \\
& <\text { false, } s>\rightarrow^{1} \text { false } \\
& \frac{A\left(a_{1}\right) s=<z_{1}, k_{1}>A\left(a_{2}\right) s=<z_{2}, k_{2}>}{<a_{1}=a_{2}, s>\rightarrow^{k_{1}+k_{2}+1} z_{1}=z_{2}} \\
& \frac{A\left(a_{1}\right) s=<z_{1}, k_{1}>A\left(a_{2}\right) s=<z_{2}, k_{2}>}{<a_{1}<a_{2}, s>\rightarrow^{k_{1}+k_{2}+1} z_{1}<z_{2}} \\
& \quad<b, s>\rightarrow^{k} t \\
& <\neg b, s>\rightarrow^{k+1} \neg t \\
& \frac{<b_{1}, s>\rightarrow^{k_{1}} t_{1} \quad<b_{2}, s>\rightarrow^{k_{2}} t_{2}}{<b_{1} \vee b_{2}, s>\rightarrow^{k_{1}+k_{2}+1} t_{1} \vee t_{2}} \\
& \frac{<b_{1}, s>\rightarrow^{k_{1}} t_{1} \quad<b_{2}, s>\rightarrow^{k_{2}} t_{2}}{<b_{1} \wedge b_{2}, s>\rightarrow^{k_{1}+k_{2}+1} t_{1} \wedge t_{2}}
\end{aligned}
$$

Theorem 3.2. B, Value and Time are functions.

Given $b$ and $s$, there exists only one $k$ and $t$ such that $\left\langle b, s>\rightarrow^{k} t\right.$.

Proof. By structural induction on $b$.

\subsection{Semantics of statements}

The set of rules corresponding to statements is based in the rules for the Structural Operational Semantics of While presented in [3]. Are new the rules for transitivity and the rules for time. As is proved after, the rules for time are equivalent to the ones presented for natural semantics in the same book.

$$
\begin{aligned}
& <a x:=a, s>\Rightarrow^{\text {Time }(a) s+1} s[a x:=\operatorname{Value}(a) s] \quad \text { ass } 1 \\
& <\text { skip, } s>\Rightarrow^{1} s \quad \text { skip } \\
& \frac{<S_{1}, s>\Rightarrow^{k}<S_{1}^{\prime}, s^{\prime}>}{<S_{1} ; S_{2}, s>\Rightarrow^{k}<S_{1}^{\prime} ; S_{2}, s^{\prime}>} \operatorname{comp} 1 \\
& \frac{<S_{1}, s>\Rightarrow^{k} s^{\prime}}{<S_{1} ; S_{2}, s>\Rightarrow^{k}<S_{2}, s^{\prime}>} \operatorname{comp} 2 \\
& \frac{\text { Value }(b) s=\text { true }}{<\text { if b then } S_{1} \text { else } S_{2}, s>\Rightarrow^{\text {Time }(b) s+1}<S_{1}, s>} \text { if } 1 \\
& \frac{\text { Value }(b) s=\text { false }}{<\text { if } b \text { then } S_{1} \text { else } S_{2}, s>\Rightarrow^{\text {Time }(b) s+1}<S_{2}, s>} \text { if } 2 \\
& <\text { while b do } S, s>\Rightarrow^{1}<\text { if } b \text { then }(S ; \text { while } b \text { do } S) \text { else skip, } s>\quad \text { while } 1 \\
& \frac{<S, s>\Rightarrow^{k_{1}}<S^{\prime}, s^{\prime}><S^{\prime}, s^{\prime}>\Rightarrow^{k_{2}}<S^{\prime \prime}, s^{\prime \prime}>}{<S, s>\Rightarrow^{k_{1}+k_{2}}<S^{\prime \prime}, s^{\prime \prime}>} \text { trans } 1
\end{aligned}
$$


$\frac{<S, s>\Rightarrow^{k_{1}}<S^{\prime}, s^{\prime}>\quad<S^{\prime}, s^{\prime}>\Rightarrow^{k_{2}} s^{\prime \prime}}{<S, s>\Rightarrow^{k_{1}+k_{2}} s^{\prime \prime}}$ trans 2

We define the semantic function

$S_{t s o s}(S) s=s^{\prime}$ in $k$ steps if and only if $<S, s>\Rightarrow^{k} s^{\prime}$

\section{Determinism}

Theorem 4.1 (The measured semantics presented in subsection 3.3 is deterministic.). If $<S, s>\Rightarrow^{k}<S^{\prime}, s^{\prime}>$ and $<S, s>\Rightarrow^{k}<S^{\prime \prime}, s^{\prime \prime}>$ then $S^{\prime}=S^{\prime \prime}$ and $s^{\prime}=s^{\prime \prime}$ and if $<S, s>\Rightarrow^{k} s^{\prime}$ and $<S, s>\Rightarrow^{k} s^{\prime \prime}$ then $s^{\prime}=s^{\prime \prime}$.

Proof. By induction on the shape of the derivation tree. If the statement is assignation, skip, if or while there is only one possible transition.

Assume the statement is $S_{1} ; S_{2}$ (composition).

- suppose $<S_{1} ; S_{2}, s>\Rightarrow^{k}<S_{3}, s^{\prime}>$ and $<S_{1} ; S_{2}, s>\Rightarrow^{k}<S_{4}, s^{\prime \prime}>$ then the form of $S_{3}$ and $S_{4}$ is $S_{3}=S_{1}^{\prime} ; S_{2}$ and $S_{4}=S_{1}^{\prime \prime} ; S_{2}$ necessarily follows that the hypothesis of the rule are $<S_{1}, s>\Rightarrow<S_{1}^{\prime}, s^{\prime}>$ or $<S_{1}, s>\Rightarrow<S_{1}^{\prime \prime}, s^{\prime \prime}>$. Then by inductive hypothesis $S_{1}^{\prime}=S_{1}^{\prime \prime}$ and $s^{\prime}=s^{\prime \prime}$.

- Suppose $<S_{1} ; S_{2}, s>\Rightarrow^{k}<S_{2}, s^{\prime}>$ and $<S_{1} ; S_{2}, s>\Rightarrow^{k}<S_{2}, s^{\prime \prime}>$ necessarily follows that the hypothesis of the rule are $\left\langle S_{1}, s\right\rangle \Rightarrow s^{\prime}$ or $\left\langle S_{1}, s\right\rangle \Rightarrow s^{\prime \prime}$. Then by inductive hypothesis $s^{\prime}=s^{\prime \prime}$.

Assume the rule applied is trans1.

- $\operatorname{suppose}<S, s>\Rightarrow^{k_{1}+k_{2}}<S_{1}, s_{1}>$ and $<S, s>\Rightarrow k_{1}+k_{2}<S_{2}, s_{2}>$. The hipothesis of the rule must be of the form $<S, s>\Rightarrow^{k_{1}}<S^{\prime}, s^{\prime}>$ and $<$ $S^{\prime}, s^{\prime}>\Rightarrow^{k_{2}}<S_{1}, s_{1}>$ or $\left\langle S^{\prime}, s^{\prime}>\Rightarrow^{k_{2}}<S_{2}, s_{2}>\right.$. Follows by the inductive hypothesis $S_{1}=S_{2}$ and $s_{1}=s_{2}$.

- suppose $<S, s>\Rightarrow^{k_{1}+k_{2}} s_{1}$ and $<S, s>\Rightarrow k_{1}+k_{2} s_{2}$. The hipothesis of the rule must be of the form $<S, s>\Rightarrow^{k_{1}}<S^{\prime}, s^{\prime}>$ and $\left\langle S^{\prime}, s^{\prime}>\Rightarrow^{k_{2}} s_{1}\right.$ or $<S^{\prime}, s^{\prime}>\Rightarrow^{k_{2}} s_{2}$. Follows by the inductive hypothesis $s_{1}=s_{2}$.

Theorem 4.2. $<S, s>\Rightarrow^{k} s^{\prime}$ and $<S, s>\Rightarrow k<S^{\prime}, s^{\prime \prime}>$ can not happen at the same time.

Proof. By induction on $k$.

- By the corresponding rule $\left\langle a s:=a, s>\rightarrow^{\text {Time }(a) s} s[a x:=\right.$ Value $(a) s]$ and is not possible $<a s:=a, s>\rightarrow^{k}<S, s^{\prime}>$ for any $k, S$ and $s^{\prime}$.

- With $<$ skip, $s>$ is similar.

- Consider the conclution of the first rule for composition: $<S_{1} ; S_{2}, s>\Rightarrow^{k}<$ $S_{1}^{\prime} ; S_{2}, s^{\prime}>$ By hypothesis $<S_{1}^{\prime} ; S_{2}, s^{\prime}>\Rightarrow^{k}<S_{2}, s^{\prime \prime \prime}>$ or $<S_{1}^{\prime} ; S_{2}, s^{\prime}>\Rightarrow<$ $S_{1}^{\prime \prime} ; S_{2}, s^{\prime \prime}>$ but not both, so $<S_{1} ; S_{2}, s>\Rightarrow<S_{2}, s^{\prime \prime \prime}>$ or $<S_{1} ; S_{2}, s>\Rightarrow<$ $S_{1}^{\prime \prime} ; S_{2}, s^{\prime \prime}>$ but not both.

- The second rule for composition is similar.

- By the if1 rule $<$ if $b$ then $S_{1}$ else $S_{2}, s>\Rightarrow^{\text {Time }(b) s+1}<S_{1}, s>$. By hypothesis $<S_{1}, s>\Rightarrow^{k} s^{\prime}$ or $\left\langle S_{1}, s>\Rightarrow^{k}<S_{1}^{\prime}, s^{\prime \prime}>\right.$ but not both. Then $<$ if $b$ then $S_{1}$ else $S_{2}, s>\Rightarrow^{\text {Time }(b) s+1+k} s^{\prime}$ or $<$ if $b$ then $S_{1}$ else $S_{2}, s>\Rightarrow^{\text {Time }(b) s+1+k}<$ $S_{1}^{\prime}, s^{\prime \prime}>$ but not both. 
- The rule if2 is likely.

- The rule while is likely.

- Consider the transitivity rules. Can happen the following cases: $<S, s\rangle \Rightarrow^{k}<$ $S^{\prime}, s^{\prime}>$ and $<S^{\prime}, s^{\prime}>\Rightarrow^{k^{\prime}}<S^{\prime \prime}, s^{\prime \prime}>$ or $<S^{\prime}, s^{\prime}>\Rightarrow^{k^{\prime}} s^{\prime \prime}$. Depending on the last transitions we chose rule trans1 or trans2. The other possibility is $<S, s>\Rightarrow^{k} s^{\prime}$ case in which this is the conclution.

Corollary 4.3. If $<S, s>\Rightarrow^{k_{1}}<S^{\prime}, s^{\prime}>$ and $<S, s>\Rightarrow^{k_{2}} s^{\prime \prime}$ then $k_{1} \leq k_{2}$.

Corollary 4.4. If $\left\langle S, s>\Rightarrow^{k_{1}}<S^{\prime}, s^{\prime}>\right.$ and $<S, s>\Rightarrow^{k_{2}} s^{\prime \prime}$ then $k_{1} \leq k_{2}$.

Proof. suppose $k_{2}<k_{1}$. Then exists states $s^{\prime}, s^{\prime \prime}$ and a statement $S^{\prime}$ such that $<S, s>\Rightarrow^{k_{2}} s^{\prime \prime}$ and $\left\langle S, s>\Rightarrow^{k_{2}}<S^{\prime}, s^{\prime}>\right.$ what by the previous theorem can not happen.

Corollary 4.5 ( $\mathrm{S}$ is a function). If $<S, s>\Rightarrow^{k_{1}} s^{\prime}$ and $<S, s>\Rightarrow^{k_{2}} s^{\prime \prime}$ then $k_{1}=k_{2}$ and $s^{\prime}=s^{\prime \prime}$.

Proof. Suppose $k_{1} \neq k 2$, in particular $k_{1}<k_{2}$. Then happens $\left.<S, s\right\rangle \Rightarrow^{k_{1}} s^{\prime}$ and $<S, s>\Rightarrow<S^{\prime}, s^{\prime}>$ what is absurd.

\section{Equivalence with the structural operational se- mantics}

\subsection{Structural operational semantics}

See [3] chapter 2 section 2 for the structural operational semantics of the language.

\subsection{Equivalence}

Lemma 5.1. If $\langle S, s\rangle \Rightarrow^{*} s^{\prime}$ then there exists $k$ such that $<S, s>\Rightarrow^{k} s^{\prime}$

Proof. The rules of the structural operational semantics are included in the measured semantics with the exception that in the last, we have, the measure of time and transitivity rules. In the structural operational semantics the transitive closure is made outside of the rules, but has the same result. If we reach a final state in the structural operational semantics, the same state is reached in the measured semantics.

Lemma 5.2. If $<S, s>\Rightarrow^{k} s^{\prime}$ then $<S, s>\Rightarrow^{*} s^{\prime}$

Proof. For each application of a rule in the measured semantics, apply the corresponding one step rule in the structural operational semantics.

Theorem $5.3\left(\mathcal{S}_{\text {sos }}=\mathcal{S}_{\text {stos }}\right)$. .

Proof. Lemmas 5.1 and 5.2 .

\section{Equivalence with the natural semantics}

\subsection{Natural semantics}

See [3] chapter 6 section 5 for the natural semantics of the language with exact execution times. 


\subsection{Equivalence}

Lemma 6.1. If $<S, s>\rightarrow^{k} s^{\prime}$ then $<S, s>\Rightarrow^{k} s^{\prime}$

Proof. By induction on the time of the derivation.

- $S=a x:=a$. We assume $\left\langle a x:=a, s>\rightarrow^{\text {Time }(a) s+1} s[\operatorname{inl}(a x):=\operatorname{inl}(\right.$ Value $[a] s)]$. Applying ass, we get $\langle a x:=a, s\rangle \Rightarrow^{\text {Time }(a) s+1} s[\operatorname{inl}(a x):=\operatorname{inl}(\operatorname{Value}(a) s)]$.

- $S=$ skip analogous.

- $S=S_{1} ; S_{2}$. Assume $<S_{1} ; S_{2}, s>\rightarrow^{k} s^{\prime \prime}$ because $\left\langle S_{1}, s>\rightarrow^{k_{1}} s^{\prime}\right.$ and $<S_{2}, s^{\prime}>\rightarrow^{k_{2}} s^{\prime \prime}$ and $k=k_{1}+k_{2}$. Applying the induction hypothesis to the derivations we get $\left\langle S_{1}, s\right\rangle \Rightarrow^{k_{1}} s^{\prime}$ and $\left\langle S_{2}, s^{\prime}\right\rangle \Rightarrow^{k_{2}} s^{\prime \prime}$. Applying rule comp 2 to $<S_{1}, s>\Rightarrow^{k_{1}} s^{\prime}$ we derive $\left\langle S_{1} ; S_{2}, s>\Rightarrow^{k_{1}}<S_{2}, s^{\prime}>\right.$, and applying trans 2 to this conclution and $\left\langle S_{2}, s^{\prime}\right\rangle \Rightarrow^{k_{2}} s^{\prime \prime}$ we get $\left\langle S_{1} ; S_{2}, s\right\rangle \Rightarrow^{k_{1}+k_{2}}$ $s^{\prime \prime}$.

- $S=$ if b then $S_{1}$ else $S_{2}$. Assume that $<$ if $b$ then $S_{1}$ else $S_{2}, s>\rightarrow^{\text {Time }(b) s+k+1}$ $s^{\prime}$, because $B[b] s=$ true and $\left\langle S_{1}, s\right\rangle \rightarrow^{k} s^{\prime}$. By induction hypothesis applied to $\left\langle S_{1}, s\right\rangle \rightarrow^{k} s^{\prime}$ we get $\left\langle S_{1}, s\right\rangle \Rightarrow^{k} s^{\prime}$. Applying if 1 to $S$ and trans 2 we get $<$ if $b$ then $S_{1}$ else $S_{2}, s>\Rightarrow^{\text {Time }(b) s+k+1} s^{\prime}$. If $B[b] s=$ false is analogous.

- $S=$ while $b$ do $S^{\prime}$. Assume that $<$ while $b$ do $S^{\prime}, s>\rightarrow^{k} s^{\prime}$ because $B[b] s=$ true and $\left\langle S^{\prime}, s>\rightarrow^{k_{1}} s^{\prime}\right.$ and $<$ while $b$ do $S^{\prime}, s^{\prime}>\rightarrow^{k_{2}} s^{\prime \prime}$. By induction hypothesis $<S^{\prime}, s>\Rightarrow^{k_{1}} s^{\prime}$ and $<$ while $b d o S^{\prime}, s^{\prime}>\Rightarrow^{k_{2}} s^{\prime \prime}$. Applying while, if 1 , and comp 2 we get:

$<$ while $b$ do $S^{\prime}, s>\Rightarrow^{\text {Time }(b) s+2}<S^{\prime} ;$ while $b$ do $S^{\prime}, s>\Rightarrow^{k_{1}}<$ while $b$ do $S^{\prime}, s^{\prime}>$ applying trans 2 two times we get $<S^{\prime}$; while $b d o S^{\prime}, s>\Rightarrow^{k_{1}+k_{2}} s^{\prime \prime}$ finally applying trans 2 to both derivations, we get $<$ while $b d o S^{\prime}, s>\rightarrow^{\text {Time }(b) s+k_{1}+k_{2}+2}$ $s^{\prime \prime}$.

- $S=$ while $b$ do $S^{\prime}$. Assume that $<$ while $b$ do $S^{\prime}, s>\rightarrow^{\text {Time }(b) s+3} s$ because $B[b] s=$ false. Then $<$ while $b$ do $S^{\prime}, s>\Rightarrow^{\text {Time }(b) s+2}<$ skip, $s>$, i.e. $<$ while $b$ do $S^{\prime}, s>\Rightarrow^{\text {Time }(b) s+3} s$.

Lemma 6.2. If $<S_{1} ; S_{2}, s>\Rightarrow^{k} s^{\prime \prime}(k \geq 2)$, then there exists a state $s^{\prime}$ and positive integers $k_{1}$ and $k_{2}$ such that $\left\langle S_{1}, s\right\rangle \Rightarrow^{k_{1}} s^{\prime}$ and $\left\langle S_{2}, s^{\prime}\right\rangle \Rightarrow^{k_{2}} s^{\prime \prime}$ where $k=k_{1}+k_{2}$.

Proof. See Lemma 2.19 in [3]. For our semantics the proof is analogous.

Lemma 6.3. If $<S, s>\Rightarrow^{k} s^{\prime}$ then $<S, s>\rightarrow^{k} s^{\prime}$

Proof. By induction on $k$.

- $S=a x:=a$. Holds because $\left\langle a x:=a, s>\rightarrow^{\text {Time }(a) s+1} s[\operatorname{inl}(a x):=\right.$ $\operatorname{inl}(\operatorname{Value}(a) s)]$.

- $S=$ skip is similar.

- $S=S_{1} ; S_{2}$. Assume that $\left\langle S_{1} ; S_{2}, s \Rightarrow^{k} s^{\prime \prime}\right.$. Applying 6.2 we know that there exists $s^{\prime}, k_{1}$ and $k_{2}$ such that $\left\langle S_{1}, s\right\rangle \Rightarrow^{k_{1}} s^{\prime}$ and $\left\langle S_{2}, s^{\prime}\right\rangle \Rightarrow^{k_{2}} s^{\prime \prime}$ where $k_{1}+k_{2}=k$. Follows $k_{1}<k$ and $k_{2}<k$.

By induction hypothesis, there are derivations in the natural semantics for $<$ $S_{1}, s>\rightarrow^{k_{1}} s^{\prime}$ and $\left\langle S_{2}, s^{\prime}>\rightarrow^{k_{2}} s^{\prime \prime}\right.$. Using the rule in the natural semantics for composition we get $<S_{1} ; S_{2}, s>\rightarrow^{k} s^{\prime \prime}$. 
- $S=$ if b then $S_{1}$ else $S_{2}$, Value(b)s $=$ true. Assume $<$ if b then $S_{1}$ else $S_{2}, s>\Rightarrow^{k}$ $s^{\prime}$, we must have transitions $<$ if $b$ then $S_{1}$ else $S_{2}, s>\Rightarrow^{\text {Time }(b) s+1}<S_{1}, s>$ and $\left\langle S_{1}, s>\Rightarrow^{k-T i m e(b) s-1} s^{\prime}\right.$. In the natural semantics we have the transition $\left\langle S_{1}, s\right\rangle \rightarrow^{k-T i m e(b) s-1} s^{\prime}$ and $<$ if b then $S_{1}$ else $S_{2}, s>\rightarrow^{k} s^{\prime}$.

The case $\operatorname{Value}(b) s=$ false is analogous.

- $S=$ while $b$ do $S^{\prime}$. Suppose we have $<$ while $b$ do $S^{\prime}, s>\Rightarrow^{k} s^{\prime}$, Value $(b) s=$ true.

The derivation in our semantics is $<$ while $b$ do $S^{\prime}, s>\Rightarrow^{1}<$ if $b$ then $\left(S^{\prime} ;\right.$ while $b$ do $\left.S^{\prime}\right)$ else skip, $s>$ and we must have a derivation for $<$ if $b$ then $\left(S^{\prime} ;\right.$ while $b$ do $\left.S^{\prime}\right)$ else skip, $s>\Rightarrow^{k-1} s^{\prime}$ that reduces to $<$ if $b$ then $\left(S^{\prime} ;\right.$ while $b$ do $\left.S^{\prime}\right)$ else skip, $s>\Rightarrow^{\text {Time }(b) s+1}<\left(S^{\prime} ;\right.$ while $b$ do $\left.S^{\prime}\right), s>$.

We have in the natural semantics

$<$ while $b$ do $S^{\prime}, s>\rightarrow^{\text {Time }(b)+t^{\prime}+t^{\prime \prime}+2} s^{\prime \prime}$, if $<S^{\prime}, s>\rightarrow^{t^{\prime}} s^{\prime}$ and $<$ while $b$ do $S^{\prime}, s^{\prime}>\rightarrow^{t^{\prime \prime}}$ $s^{\prime \prime}$,

$<$ if $b$ then $\left(S^{\prime} ;\right.$ while $b$ do $\left.S^{\prime}\right)$ else skip, $s>\rightarrow^{\text {Time }(b) s+t+1} s^{\prime}$ where $t=t^{\prime}+t$ and $<\left(S^{\prime} ;\right.$ while $b$ do $\left.S^{\prime}\right), s>\rightarrow^{t} s^{\prime}$. By Lemma $6.3<\left(S^{\prime} ;\right.$ while $b$ do $\left.S^{\prime}\right), s>\Rightarrow^{t}$ $s^{\prime}$, then $k-1=1+t+\operatorname{Time}(b) s$

follows $k-1=$ Time $(b) s+t+1$ then $k=$ Time $(b) s+t+2$.

- $S=$ while $b$ do $S^{\prime}$. Suppose we have $<$ while $b$ do $S^{\prime}, s>\Rightarrow^{k} s$,Value $(b) s=$ false.

The derivation in our semantics is $<$ while $b$ do $S^{\prime}, s>\rightarrow^{1}<$ if $b$ then $\left(S^{\prime} ;\right.$ while $b$ do $\left.S^{\prime}\right)$ else skip, $s>$

And we must have a derivation for $<$ if b then $\left(S^{\prime} ;\right.$ while b do $\left.S^{\prime}\right)$ else skip, $s>\Rightarrow^{k-1} s$.

We have in the natural semantics $<$ if $b$ then $\left(S^{\prime}\right.$; while $b$ do $\left.S^{\prime}\right)$ else skip, $s>\rightarrow^{\text {Time }(b) s+2} s$, where $<$ skip, $s>\rightarrow^{1} s$ and $k-1=$ Time(b) $s+2$ then it follows $<$ while $b$ do $S^{\prime}, s>\rightarrow^{k}$ $s$.

Theorem 6.4 $\left(\mathcal{S}_{t n s}=\mathcal{S}_{t s o s}\right)$. .

Proof. Lemmas 6.1 and 6.3 .

\section{Conclusions and further work}

We have presented a semantics that takes account of the number of steps of evaluation of expressions and statements following traditional complexity theory.

We expect in a future work, extend the semantics presented in this paper with functions and procedures. We are interested in finding recurrence expressions to measure the time of iterative and recursive programs. Recursivity will give rise to recurrences and in this way can be possible to find ecuations for the time of a program. 


\section{References}

[1] Matthew Hennesy. The Semantics of Programming Languages, An Elementary Introduction using Structural Operational Semantics, John Wiley \& Sons, Chichester-New york-Brisbane-Toronto-Singapore, 1990.

[2] Gilles Kahn. Natural Semantics, Programming of Future Generation Computers, Elsevier Science Publishers (North-Holland), 1988.

[3] H.R.Nielson and F.Nielson. Semantics with Applications, A Formal Introduction for Computer Science, John Wiley \& Sons, 1992.

[4] Gordon Plotkin. A structural Approach to operational semantics, Technical Report DAIMI FN-19, Aarhus University,1981. 